\title{
TIPE2 functions as a metastasis suppressor via negatively regulating $\beta$-catenin through activating GSK3 $\beta$ in gastric cancer
}

\author{
JIE WU $^{1 *}$, HAITAO ZHANG ${ }^{2 *}, \mathrm{CHUN} \mathrm{XU}^{1}$, HONG XU ${ }^{1}$, \\ XIUMIN ZHOU ${ }^{1}$, YUFENG XIE ${ }^{1}$ and MIN TAO $^{1}$ \\ Departments of ${ }^{1}$ Oncology and ${ }^{2}$ General Surgery, The First Affiliated Hospital \\ of Soochow University, Suzhou, Jiangsu 215006, P.R. China
}

Received August 15, 2015; Accepted October 12, 2015

DOI: 10.3892/ijo.2015.3224

\begin{abstract}
Tumor necrosis factor (TNF)- $\alpha$-induced protein 8-like 2 (TNFAIP8L2, TIPE2) is a novel anti-inflammatory factor involved in maintaining immune homeostasis. Accumulating evidence has also shown that TIPE2 displays tumor-suppressive effects in several tumor types. Previous studies revealed that TIPE2 inhibits hepatocellular carcinoma metastasis by repressing Ral and Rac1 GTPases. However, its antimetastatic activity and underlying mechanism in other human cancers is largely unknown. We investigated TIPE2 in AGS, HGC-27 and SGC-7901 human gastric cancer cells compared with GES-1 normal human gastric mucous epithelial cells. We demonstrated that TIPE2 was expressed in GES-1 gastric mucous epithelial cells but lost in all three types of gastric cancer cells. We then performed a gain-offunction study by adenovirus-mediated TIPE2 overexpression (AdVTIPE2) and investigated the effects of TIPE2 on migration and invasion of AGS human gastric cancer cells. Wound healing and Transwell invasion assays showed that forced expression of TIPE2 markedly suppressed the gastric cancer cell migration and invasion in vitro. Mechanistically, TIPE2 remarkably reduced the total levels of pAKT, pGSK3 $\beta$ and $\beta$-catenin as well as the nuclear level of $\beta$-catenin in gastric cancer cells. The TIPE2-elicited antimetastatic effect in gastric cancer was closely associated with the inhibition of AKT signaling and enhancement of GSK3 $\beta$ activity followed by the degradation and decreased translocation to nucleus
\end{abstract}

Correspondence to: Dr Min Tao or Professor Yufeng Xie, Department of Oncology, The First Affiliated Hospital of Soochow University, 188 Shizi Street, Suzhou, Jiangsu 215006, P.R. China

E-mail:mtao@medmail.com.cn

E-mail:sdxyf@163.com

${ }^{*}$ Contributed equally

Key words: gastric cancer, tumor necrosis factor- $\alpha$-induced protein 8 -like 2 , metastasis, glycogen synthase kinase $3 \beta$, $\beta$-catenin of $\beta$-catenin. These results provide the first compelling evidence that TIPE2 suppresses gastric cancer metastasis via downregulating $\beta$-catenin signaling through inhibiting AKT and activating GSK $3 \beta$, indicating that TIPE2 is a promising therapeutic target for human gastric cancer metastasis.

\section{Introduction}

Tumor necrosis factor (TNF)- $\alpha$-induced protein 8 -like 2 (also referred to as TNFAIP8L2, TIPE2) is a newly described anti-inflammatory factor in an experimental autoimmune encephalomyelitis (EAE) mouse model, and an important member of the TIPE family including TNFAIP8, TIPE1, TIPE2 and TIPE3 the four known members (1,2). TIPE2 as a novel death effector domain (DED) like domain-containing immune negative molecule is crucial for homeostasis maintenance in both the adaptive and innate immunity $(1,3)$. TIPE2-deficient mouse displays multiple organ inflammation, splenomegaly, leukocytosis and hyper-responsiveness to septic shock, resulting in premature death (1). TIPE2-deleted macrophages and $\mathrm{T}$ cells are hyper-responsive to Toll-like receptor (TLR) and T cell receptor (TCR) activation and drastically secrete inflammatory cytokines (1). TIPE2 inhibits TLR and TCR signaling via suppressing activation of activating protein-1 (AP-1), nuclear factor- $\kappa \mathrm{B}(\mathrm{NF}-\kappa \mathrm{B})$, c-Jun N-terminal kinase (JNK) and p38 MAPK while facilitating Fas-triggered T cell apoptosis (1). TIPE2 also control macrophage innate immunity to bacterial infection by negatively regulating phagocytosis and oxidative burst through directly interacting with and blocking Rac GTPases (4). Moreover, TIPE2 negatively modulates dendritic cell (DC) innate immunity to RNA viral infection by inhibiting phosphatidylinositol-3-kinase (PI3K)-Rac pathway (5). Additionally, TIPE2 restrains inducible nitric oxide synthase (iNOS) and NO generation by arginine metabolism switch from nitric oxide synthase to arginase, leading to inflammation suppression (6). Notably, TIPE2 maintains immunosuppressive property of $\mathrm{CD} 4{ }^{+} \mathrm{CD} 25^{+}$ regulatory $\mathrm{T}$ cells via orchestrating expression of immunosuppressive molecules such as cytotoxic T-lymphocyte-associated protein 4 (CTLA4), forkhead box P3 (Foxp3), TGF- $\beta$ and IL-10 (7). 
The cell-autonomous role of TIPE2 in human cancer is receiving increasing attention. A seminal study (8) demonstrated that TIPE2 can act as a novel inhibitor of oncogenic Ras, and dramatically suppresses cell survival and motility via attenuating activation of downstream signaling molecules AKT and Ral GTPase by competitively binding to Ras-interacting domain (RID) of Ras effector RGL and preventing Ras/RGL active complex formation. Overexpression of TIPE2 initiates hepatocellular carcinoma (HCC) death and retards Ras-induced tumorigenesis $(8,9)$. TIPE2 also suppresses HCC metastasis by inhibiting Racl GTPase and subsequently reducing polymerization of F-actin, matrix metallopeptidase 9 (MMP9) and urokinase plasminogen activator (uPA) (9). Furthermore, TIPE2 has been found to be markedly downregulated or lost in human HCC clinical tissues, which is significantly associated with tumor metastasis $(8,9)$. Subsequent studies also showed that TIPE2 is frequently reduced or absent in human gastric cancer and lung cancer tissues, contributing to poor prognosis (10-12). Forced expression of TIPE2 inhibits tumor cell growth and induces cell cycle alteration and apoptosis by upregulating expression of cyclin-dependent kinase (CDK) inhibitors and apoptosis-related molecules (10-12). These reports indicated that TIPE2 is capable of exerting tumor-suppressive effects in a cell-autonomous manner.

Gastric cancer is one of the most common cancers worldwide and the leading cause of cancer-related deaths (13). Metastatic gastric cancer is a life-threatening disease with no effective treatment. Thus, deep understanding of the mechanism underlying gastric cancer metastasis and discovery of novel treatment targets is urgently needed. Accumulating evidence has demonstrated that TIPE2 exhibits tumorsuppressive effects in several tumor types (8-12). Clinical data have also shown that TIPE2 is reduced in human gastric cancer tissues (10). However, its effects on gastric cancer metastasis are largely unclear. In the present study, we analyzed TIPE2 expression in a panel of human gastric cancer cells, examined the effects of TIPE2 on human gastric cancer cell migration and invasion in vitro by adenovirusmediated TIPE2 overexpression, and elucidated its molecular mechanism.

\section{Materials and methods}

Vectors, cell lines and antibodies. The marker gene green fluorescent protein (GFP)-expressing pAdTrack-CMV (14) adenoviral transfer plasmid, BJ5183 bacteria and QBI-293A human embryonic kidney cell line for adenovirus packaging and amplification were kindly provided by Professor Jiang Zhong (Department of Microbiology, College of Life Science, Fudan University, Shanghai, China). The integrin-binding motif Arg-Gly-Asp (RGD)-modified pAdEasy-1 (Ad5E1/E3-deleted) adenoviral backbone plasmid (15) was kindly provided by Professor Jim Xiang (Saskatoon Cancer Agency, Saskatoon, SK, Canada). The AGS, HGC-27 and SGC-7901 human gastric cancer cell lines and GES-1 human gastric mucous epithelial cell line were purchased from the Cell Bank, Type Culture Collection of Chinese Academy of Sciences (Shanghai, China). The goat anti-TIPE2 (C-18) antibody and secondary antibodies such as anti-goat IgG-horseradish peroxidase (HRP), antirabbit IgG-HRP and anti-rabbit IgG-phycoerythrin (PE) were purchased from Santa Cruz Biotechnology (Shanghai, China). The rabbit antibodies specific for pAKT (244F9), AKT (11E7), pGSK3 $\beta$ (D3A4), GSK3 $\beta$ (27C10), $\beta$-catenin (D10A8), $\beta$-actin (13E5) and histone $\mathrm{H} 3$ (3H1) were purchased from Cell Signaling Technology (Danvers, MA, USA).

Reverse transcription (RT)-PCR assay. The endogenous TIPE2 expression in human gastric cancer cells was evaluated by RT-PCR analysis. The total RNAs were extracted from AGS, HGC-27 and SGC-7901 human gastric cancer cells ( $2 \times 10^{6}$ cells/each) and GES-1 normal human gastric mucous epithelial cells $\left(2 \times 10^{6}\right.$ cells) using MiniBEST universal RNA extraction kit (Takara, Dalian, Liaoning, China) and firststrand cDNAs were synthesized from RNAs using reverse transcriptase MuMLV (Thermo Fisher Scientific, Shanghai, China). The PCR reactions were then performed using cDNAs as templates and primers (Sangon Biotechnology Inc., Shanghai, China) specific for human TIPE2 (TIPE2-F1, 5'-gac tga cca cat acc cca ctc-3' and TIPE2-R1, 5'-tca cca aag cta agt gcc gt-3' for amplifying $348 \mathrm{bp}$; TIPE2-F2, 5'-cag tga ctg acc aca tac ccc-3' and TIPE2-R2, 5'-tgg cca ctt tga tca ggt cc-3' for amplifying $237 \mathrm{bp}$ ) or the housekeeping gene $\beta$-actin ( $\beta$-actin-F, 5'-tgc gtg aca tta agg aga ag-3' and $\beta$-actin-R, $5^{\prime}$-ctg cat cet gtc ggc aat g-3' for amplifying $317 \mathrm{bp}$ ) (an internal control). The human TIPE2 cDNA fragment containing full-length coding sequence (CDS) (GenBank accession no. NM_024575) was amplified from human peripheral blood mononuclear cells (PBMCs) $\left(5 \times 10^{6}\right.$ cells) using TIPE2specific primers (TIPE2-F3, 5'-gtg act gac cac ata ccc ca-3' and TIPE2-R3, 5'-agt gtt agt gcc agg tga gc-3' for amplifying $684 \mathrm{bp}$ ) by RT-PCR, subcloned into pMD19-T cloning vector (Takara) and sequenced as previously described (16). The adenovirus-mediated exogenous human TIPE2 transcriptional expression was also analyzed by RT-PCR using TIPE2 CDS-specific primers (TIPE2-F4, 5'-acc gtc gac gcc acc atg gag tcc ttc agc tca aag-3' and TIPE2-R4, 5'-gca ctc gag tca gag ctt ccc ttc gtc tag cag c-3' for amplifying $555 \mathrm{bp}$ ).

Recombinant adenovirus AdVTIPE2 construction. The human TIPE2 CDS fragment (555 bp) was amplified by PCR using pMD19-T-TIPE2 plasmids as templates and primers (TIPE2F4, 5'-acc gtc gac gec acc atg gag tcc ttc agc tca aag-3' and TIPE2-R4, 5'-gca ctc gag tca gag ctt ccc ttc gtc tag cag c-3'), and inserted into GFP marker gene-expressing pAdTrackCMV adenoviral transfer plasmid between SalI and XhoI sites to form pAdTrack-CMV-TIPE2. To develop a more efficient gene delivery system, we used Arg-Gly-Asp (RGD)modified and Ad5E1/E3-deleted pAdEasy-1 which contains an integrin-binding motif RGD sequence in the HI loop of adenoviral fiber as an adenoviral backbone plasmid (15). Based upon the homologous recombination of pAdTrack-CMVTIPE2 or pAdTrack-CMV with RGD-modified pAdEasy-1 in BJ5183 bacteria, the RGD-modified replication-incompetent AdVTIPE2 and AdV (blank adenovirus control) adenoviruses expressing GFP were subsequently generated and amplified in QBI-293A cells as reported previously (14), and further purified by cesium chloride $(\mathrm{CsCl})$ density-gradient ultracentrifugation.

Fluorescence microscopic analysis. The titer of AdVTIPE2 and $\mathrm{AdV}$ adenoviruses was determined using gene transfer unit 
(GTU) method by calculating the number of GFP-expressing QBI-293A cells within $18 \mathrm{~h}$ after adenoviral infection by fluorescence microscopy. Accordingly, the ratio of infectious adenovirus (GTU) to target cells is called multiplicity of infection (MOI). To select an optimal MOI for a maximal adenoviral infection and human TIPE2 transgene expression in tumor cells, the AGS human gastric cancer cells $\left(1 \times 10^{4}\right.$ cells $/ 200 \mu$ l culture medium, i.e., RPMI-1640 medium supplemented with $10 \%$ fetal bovine serum) (Hyclone, Logan, UT, USA) were plated into 96-well plates overnight and treated with AdVTIPE2, AdV or PBS without adenovirus at various MOIs $(0,10,25,50,100$ or 200) in culture medium. Twenty-four hours after infection, the GFP expression was observed and photographed at x200 higher-power field by fluorescence microscopy and the adenoviral infection efficiency was analyzed.

Wound healing assay. The effect of adenovirus-mediated TIPE2 overexpression on gastric cancer cell migration in vitro was analyzed by wound healing assay. Briefly, the outer bottom of 6-well culture plates was marked to be used as a reference point during image acquisition. The AGS human gastric cancer cells were plated to $70 \%$ confluence as a monolayer in marked 6-well plates and treated with AdVTIPE2 or AdV at the optimal MOI of 10. The AdVTIPE2- or AdV-infected AGS tumor cells were termed AGS-TIPE2 and AGS-Mock, respectively. Twenty-four hours after infection, scratches were created in the monolayer with a pipette tip and the wells were gently washed with fresh medium to remove the detached cells. Progression of tumor cell migration was observed and photographed by microscopy (x100 low-power field) at the beginning and at $24 \mathrm{~h}$ after wounding. The gap distance was then quantitatively calculated using Image J software (National Institutes of Health, Bethesda, MD, USA) and the migratory ability of tumor cells was analyzed.

Transwell invasion assay. The effect of adenovirus-mediated TIPE2 overexpression on gastric cancer cell invasion in vitro was assessed by Transwell invasion assay. Briefly, the $12.5 \mu \mathrm{l}$ Matrigel (50 mg/l) (BD Biosciences, Shanghai, China) was diluted in $87.5 \mu \mathrm{l}$ serum-free RPMI-1640 medium. The $100 \mu \mathrm{l}$ Matrigel diluted solution was added to 24-well Transwell chamber (Corning Inc., Corning, NY, USA) that incorporated a polycarbonate filter membrane with a diameter of $6.5 \mathrm{~mm}$ and a pore size of $8 \mu \mathrm{m}$, dried in a laminar hood overnight, and reconstituted in $100 \mu \mathrm{l}$ serum-free RPMI-1640 medium for $2 \mathrm{~h}$. The AGS-TIPE2 and AGS-Mock tumor cells ( $2 \times 10^{5}$ cells $/ 100 \mu \mathrm{l}$ serum-free RPMI-1640 medium) were added to the upper chamber of the Transwell. The lower chamber was filled with $600 \mu \mathrm{l}$ of culture medium. After 24-h incubation, tumor cells on the upper surface of the filter were removed using a cottons wab and cells invading into the bottom side of the insert were fixed by $4 \%$ paraformaldehyde, stained with crystal violet, photographed and quantified by counting them in 5 random $\times 200$ high-power fields. The invasive ability of tumor cells was then analyzed.

Western blot analysis. The AGS human gastric cancer cells $\left(1 \times 10^{7}\right.$ cells) were treated with AdVTIPE2 (10 MOI) or AdV (10 MOI). After 24-h treatment, the AGS-TIPE2 and AGS-Mock tumor cells were collected, washed with cold PBS and lysed in lysis buffer $\left(1 \times 10^{7}\right.$ cells $/ 1 \mathrm{ml}$ lysis buffer) for preparation of total cellular proteins using mammalian cell lysis kit (SigmaAldrich, Shanghai, China). The nuclear proteins derived from AGS-TIPE2 and AGS-Mock tumor cells were also isolated using nuclear extraction kit (Sigma-Aldrich, Shanghai, China). The total and nuclear proteins (100 $\mu \mathrm{g} /$ lane) were resolved by $12 \%$ sodium dodecyl sulfate-polyacrylamide gel electrophoresis (SDS-PAGE), transferred onto polyvinylidene difluoride (PVDF) membranes (Merck Millipore, Shanghai, China) and then subjected to western blot analysis of TIPE2, pAKT, AKT, pGSK $3 \beta$, GSK $3 \beta, \beta$-catenin, $\beta$-actin (a total protein loading control); $\beta$-catenin, histone H3 (a nuclear protein loading control), respectively. The membranes were developed using SuperEnhanced chemiluminescence detection kit (Applygen Technology Inc., Beijing, China) and the protein bands were visualized after their exposure to Kodak X-ray film.

Confocal microscopic analysis. The AGS human gastric cancer cells were cultured in chamber slides (Sigma-Aldrich, Shanghai, China) and treated with AdVTIPE2 (10 MOI) or AdV (10 MOI) for $24 \mathrm{~h}$. The AGS-TIPE2 and AGS-Mock tumor cells were fixed in $4 \%$ paraformaldehyde for $20 \mathrm{~min}$, washed 3 times with PBS, permeabilized in $0.2 \%$ Triton X-100, blocked with 5\% normal goat serum and incubated with rabbit anti- $\beta$ catenin primary antibody for $1 \mathrm{~h}$. The slides were washed and stained with PE-conjugated anti-rabbit IgG secondary antibody. The slides were then washed and prevented from fading using Prolong diamond antifade with DAPI (Life Technology, Shanghai, China). The $\beta$-catenin expression in AGS tumor cells was observed by confocal microscopy.

Statistical analysis. The data are presented as the mean \pm standard deviation (SD) and statistically processed by Student's t-test for comparison of differences between two groups using SPSS 10.0 software (SPSS, Chicago, IL, USA). A value of $\mathrm{p}<0.05$ was considered statistically significant.

\section{Results}

TIPE2 expression is absent in gastric cancer. Previous studies showed that TIPE2 is not expressed or poorly expressed in most human cancer cells (17). To further examine TIPE2 expression in human gastric cancer cells, we assessed TIPE2 mRNA in a panel of human gastric cancer cell lines including AGS, HGC-27 and SGC-7901 by RT-PCR analysis. In contrast to GES-1 normal human gastric mucous epithelial cells, TIPE2 expression was almost undetectable in all the three human gastric cancer cells using two TIPE2-specific primer pairs for amplifying either 348 or 237 bp human TIPE2 cDNA (Fig. 1A). Consistent with our results, TIPE2 is also absent in U-87 MG and U251 brain tumor cells (17). A recent study (10) revealed that TIPE2 is markedly decreased or undetectable in gastric cancer tissues compared with adjacent control tissues. Our cellular model data together with the clinical data strongly indicated that TIPE2 is almost completely lost in human gastric cancer, which may be connected to gastric cancer progression.

Adenovirus-directed TIPE2 overexpression in gastric cancer. To decipher the pathological role of TIPE2 dysregulation in 
A

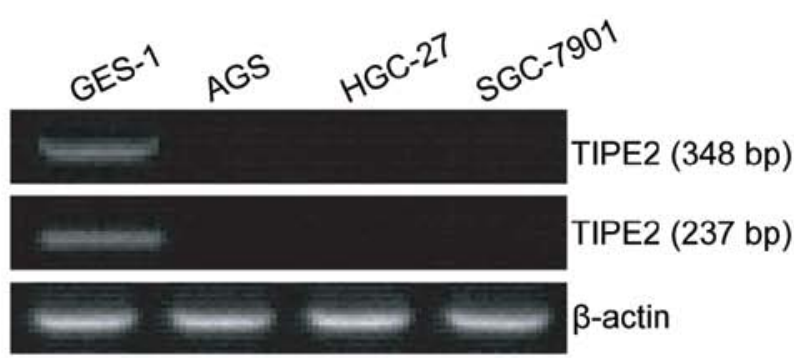

B

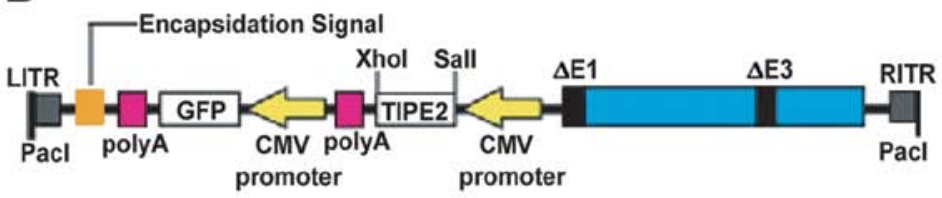

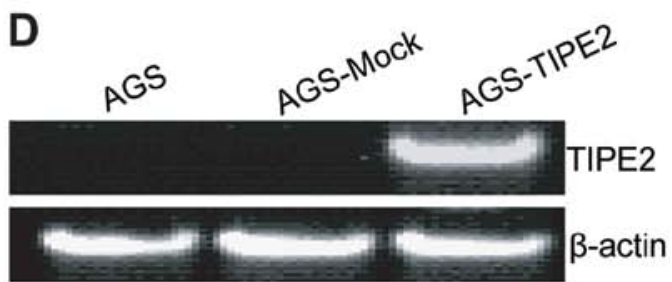

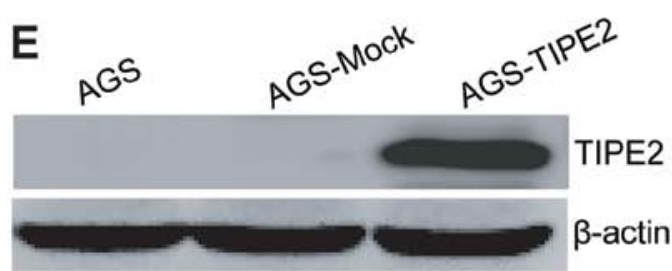

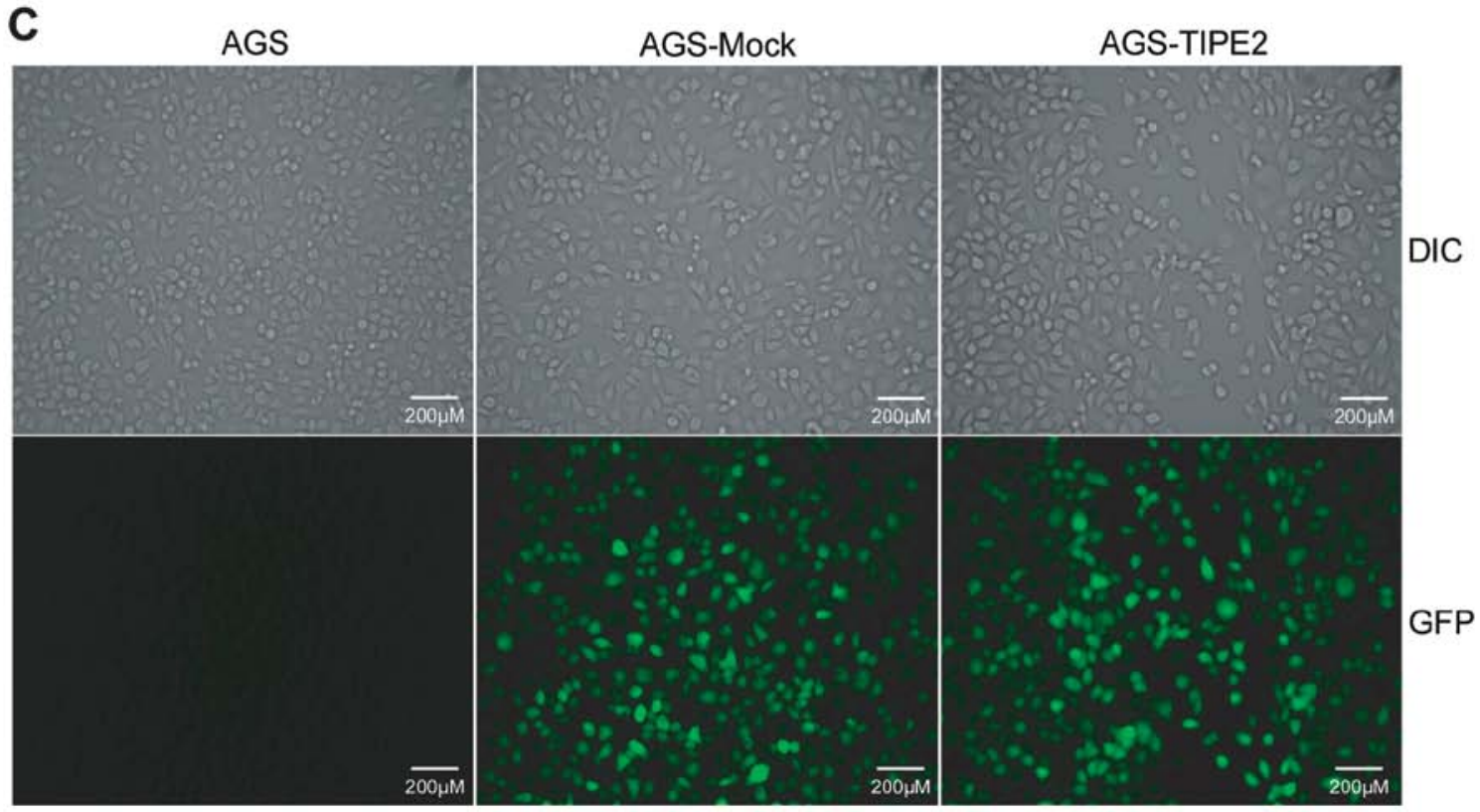

Figure 1. Adenovirus-mediated TIPE2 gene transfer in gastric cancer cells. (A) RT-PCR analysis of endogenous human TIPE2 expression in gastric cancer cells. Total RNAs were extracted from AGS, HGC-27 and SGC-7901 human gastric cancer cells and GES-1 human gastric mucous epithelial cells. The firststrand cDNAs were synthesized from RNAs using reverse transcriptase; PCRs were conducted using primer sets specific for human TIPE2 (348 or 237 bp cDNA) and housekeep gene $\beta$-actin, respectively. (B) AdVTIPE2 construction strategy. The human TIPE2 555 bp CDS fragment was amplified by PCR using pMD19-T-TIPE2 plasmids as templates and subcloned into pAdTrack-CMV adenoviral transfer plasmid between SalI and XhoI sites, where TIPE2 transcription is under control of CMV $\left({ }^{\mathrm{st}} \mathrm{CMV}\right)$ promoter, while GFP transcription is under control of CMV $\left(2^{\text {nd }} \mathrm{CMV}\right)$ promoter. The AdVTIPE2 adenovirus was then generated by homologous recombination with RGD-modified and Ad5E1/E3-deleted pAdEasy-1 in BJ5183 bacteria followed by packaging/amplification in QBI-293A cells. CMV, cytomegalovirus promoter; GFP, green fluorescent protein; polyA, SV40 polyadenylation signal; LITR, left-hand inverted terminal repeat; RITR, right-hand inverted terminal repeat. (C) Representative images of differential interference contrast (DIC) and GFP. The AGS tumor cells were infected with AdVTIPE2 or AdV at the MOI of 10 for $24 \mathrm{~h}$, and then observed under GFP fluorescence and DIC images by fluorescence microscopy. (D) RT-PCR analysis of adenovirus-mediated TIPE2 transcriptional expression. Total RNAs were extracted from AdVTIPE2- or AdV-infected (AGS-TIPE2 or AGS-Mock) and uninfected AGS tumor cells. The first-strand cDNAs were synthesized from RNAs using reverse transcriptase; PCRs were conducted using primer sets specific for human TIPE2 (555 bp CDS) and housekeeping gene $\beta$-actin, respectively. (E) Western blot analysis of adenovirus-mediated TIPE2 translational expression. Total cellular proteins derived from AdVTIPE2- or AdV-infected (AGS-TIPE2 or AGS-Mock) and uninfected AGS tumor cells were immunoblotted with anti-TIPE2 and anti- $\beta$-actin antibody, respectively. Data shown are representative of three independent experiments.

human gastric cancer, we cloned the human TIPE2 CDS by RT-PCR from PBMCs and constructed the RGD-modified replication-deficient recombinant adenovirus expressing human TIPE2 and marker gene GFP (AdVTIPE2) (Fig. 1B). To select an optimal MOI for a maximal adenovirus-mediated TIPE2 expression, but a minimal adenovirus-induced cytotoxic effect, the AGS human gastric cancer cells were infected with GFP-expressing AdVTIPE2 or AdV at different MOIs for $24 \mathrm{~h}$ and then examined by fluorescence microscopy.
More than 90\% of GFP expression was found in AdVTIPE2or AdV-infected AGS tumor cells at a MOI of 10 (Fig. 1C) or above (data not shown). In addition, there was almost no adenovirus-elicited cytotoxicity in 10 MOI blank adenovirus AdV-infected AGS tumor cells compared to uninfected control AGS tumor cells (Fig. 1C). RT-PCR (Fig. 1D) and western blot (Fig. 1E) analysis further showed that adenovirusmediated TIPE2 exogenous gene was abundantly expressed at both the transcriptional and translational levels in $10 \mathrm{MOI}$ 
A

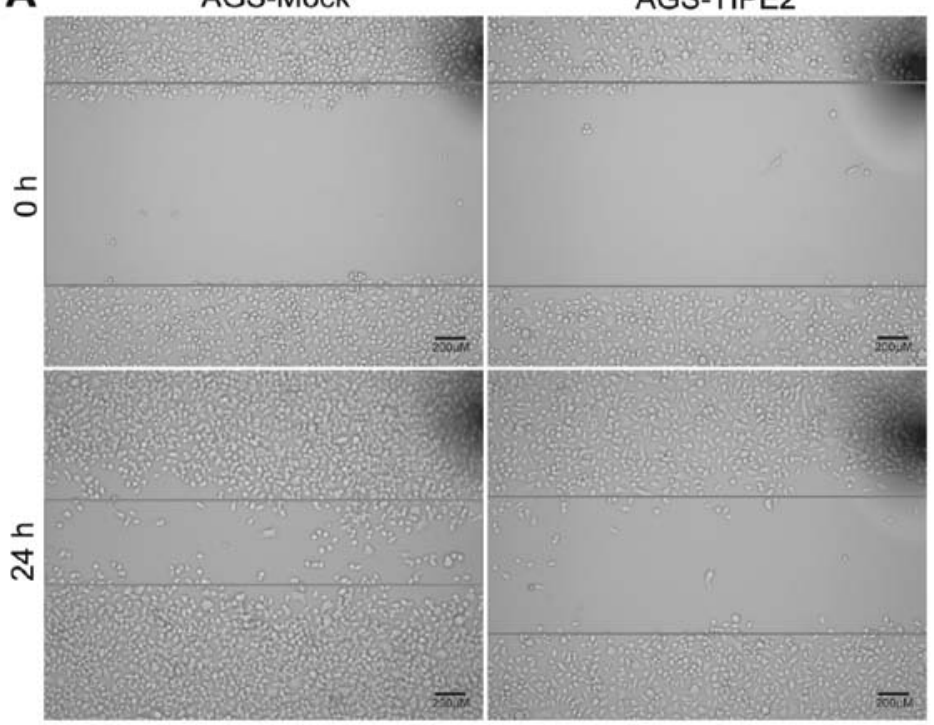

C

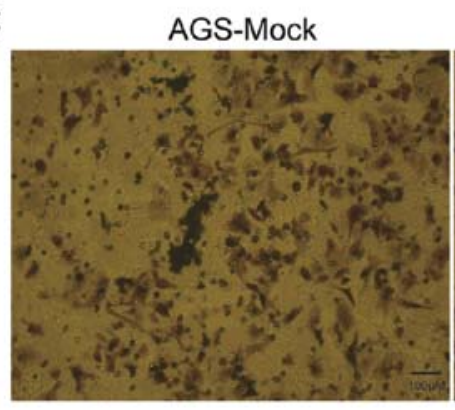

AGS-TIPE2

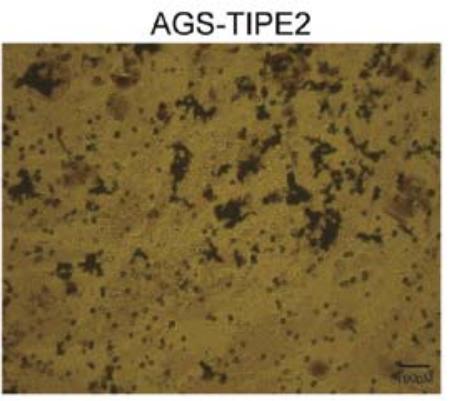

B
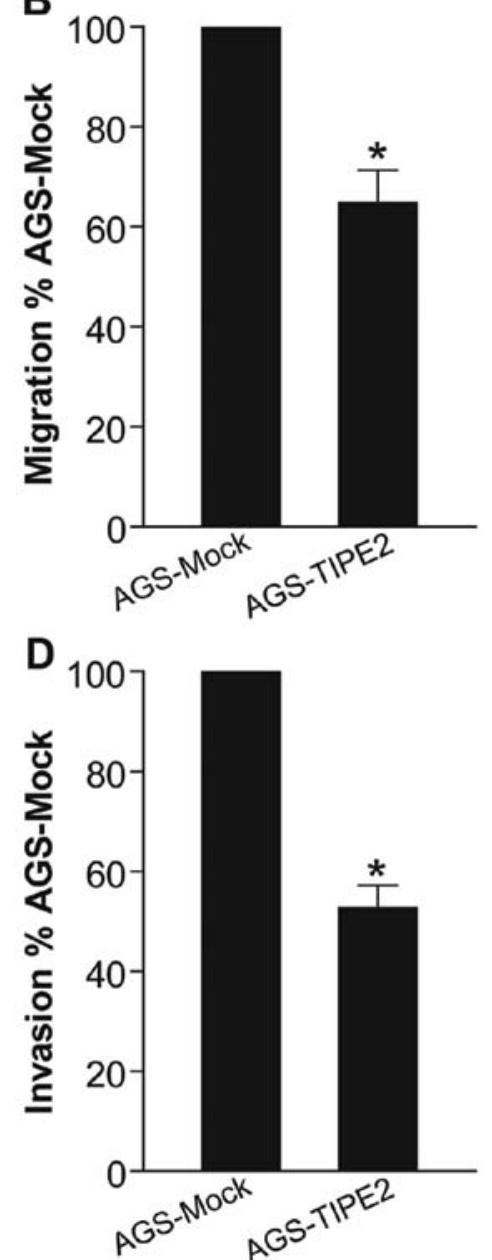

Figure 2. AdVTIPE2 inhibits gastric cancer migration and invasion in vitro. The AdVTIPE2- or AdV-infected AGS tumor cells (AGS-TIPE2 or AGS-Mock) were scratched in the monolayer with a pipette tip. Wound closures were photographed at 0 and $24 \mathrm{~h}$ after wounding. The representative images of wound healing assay of the indicated cells are shown (A). The gap distance was then quantitatively analyzed using ImageJ software and the relative migratory ability of tumor cells was calculated by comparison with AGS-Mock group (B). " $\mathrm{p}<0.05$, Student's t-test, $\mathrm{n}=3$ replicates per condition. The AGS-TIPE2 or AGS-Mock tumor cells were added to Matrigel-coated 24-well Transwell upper chamber. The lower chamber was filled with $600 \mu 1$ of culture medium. After $24-\mathrm{h}$ incubation, tumor cells on the upper surface of the filter were removed and cells invading into the bottom side of the insert were fixed by $4 \%$ paraformaldehyde, stained with crystal violet, photographed and counted. The representative images of Transwell invasion assay of the indicated cells are shown (C). The relative invasive ability of tumor cells was calculated by comparison with AGS-Mock group (D). ${ }^{*} \mathrm{p}<0.05$, Student's t-test, $\mathrm{n}=3$ replicates per condition, $\mathrm{n}=5$ observations per replicate. Data shown are representative of three independent experiments.

AdVTIPE2-infected AGS tumor cells, but not in AdV-infected or uninfected control cells. These data suggested that $10 \mathrm{MOI}$ can be employed as an optimal infection dose for adenovirusmediated TIPE2 gene transfer and overexpression in AGS tumor cells.

TIPE2 suppresses gastric cancer migration and invasion. Tumor metastasis is a key hallmark of cancer, causing as much as $90 \%$ of cancer-associated deaths $(18,19)$. TIPE2 has been found to inhibit hepatocellular carcinoma metastasis $(8,9)$. To investigate the effect of TIPE2 on gastric cancer migration and invasion in vitro, we carried out a gain-of-function study by adenovirus-mediated TIPE2 gene transfer into AGS human gastric cancer cells and performed wound healing and Transwell chamber invasion assays. As shown in Fig. 2A and B, adenovirus-mediated TIPE2 overexpression obviously inhibited the migratory speed of AGS gastric cancer cells compared with that of AdV-infected AGS-Mock tumor cells $(\mathrm{p}<0.05)$. Furthermore, TIPE2 overexpression also remarkably suppressed the invasive ability of AGS tumor cells (Fig. 2C and D) $(\mathrm{p}<0.05)$. Our data for the first time demonstrated that TIPE2 negatively modulated gastric cancer cell motility and invasion.

TIPE2 downregulates $\beta$-catenin signaling via inhibiting $A K T$ and activating GSK $3 \beta$. To further address the molecular mechanism responsible for TIPE2-mediated inhibition of gastric cancer cell migration and invasion, the expression of pAKT, AKT, pGSK3 $\beta$, GSK3 $\beta$ and $\beta$-catenin in AdVTIPE2and AdV-infected AGS human gastric cancer cells was determined by western blot analysis. As shown in Fig. 3A and $\mathrm{B}$, the total levels of pAKT, pGSK $3 \beta$ and $\beta$-catenin in AGS-TIPE2 tumor cells was markedly reduced compared with that in AGS-Mock tumor cells. Moreover, the nuclear level of $\beta$-catenin in AGS-TIPE2 tumor cells was also clearly decreased. Confocal microscopic analysis (Fig. 3C) further 

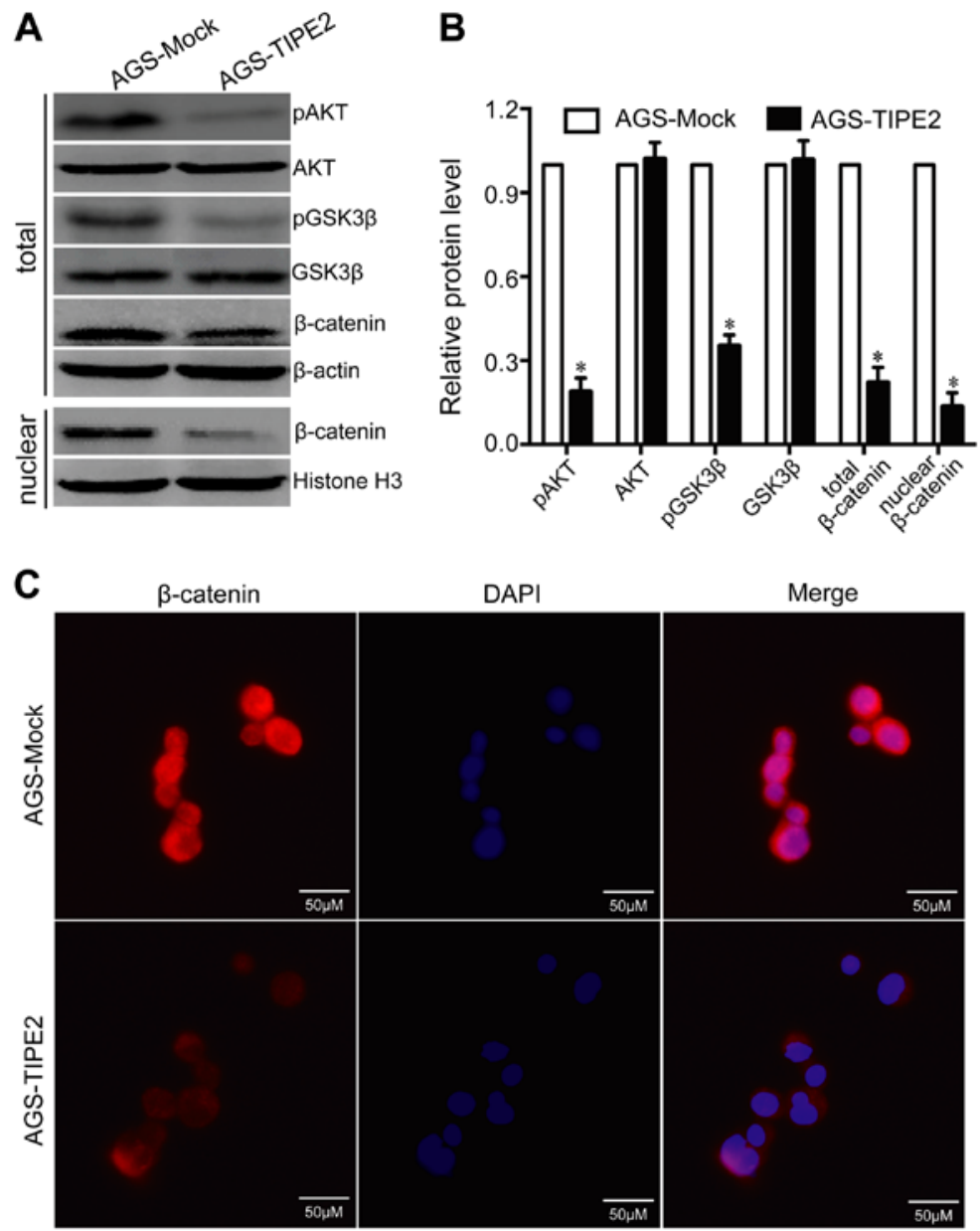

Figure 3. AdVTIPE2 suppresses gastric cancer metastasis via downregulating $\beta$-catenin through inhibiting AKT and activating GSK3 $\beta$. (A and B) Western blot analysis of AKT, GSK3 $\beta$ and $\beta$-catenin. The total cellular proteins derived from AdVTIPE2- and AdV-infected AGS tumor cells (AGS-TIPE2 or AGS-Mock) were immunoblotted with a panel of antibodies specific for pAKT, AKT, pGSK3 $\beta$, GSK3 $\beta, \beta$-catenin and $\beta$-actin; the nuclear proteins were immunoblotted with anti- $\beta$-catenin and anti-histone $\mathrm{H} 3$ antibody, respectively. The representative images of western blot analysis are shown (A). The expression of each index was normalized to expression level of $\beta$-actin (total cellular proteins) or histone $\mathrm{H} 3$ (nuclear proteins), and the relative change was expressed as a ratio or fold, with 1 being the value for AGS-Mock group (B). " $p<0.05$, Student's t-test, $n=3$ replicates per condition, $n=3$ replicates per sample. (C) Confocal microscopic analysis of $\beta$-catenin immunofluorescence. The AGS-TIPE2 and AGS-Mock tumor cells were fixed in $4 \%$ paraformaldehyde, permeabilized in $0.2 \%$ Triton X-100, blocked with 5\% normal goat serum and incubated with anti- $\beta$-catenin primary antibody followed by incubation with PE-labeled secondary antibody, and then observed under confocal microscopy. The representative images of confocal microscopic analysis are shown. Data shown are representative of three independent experiments.

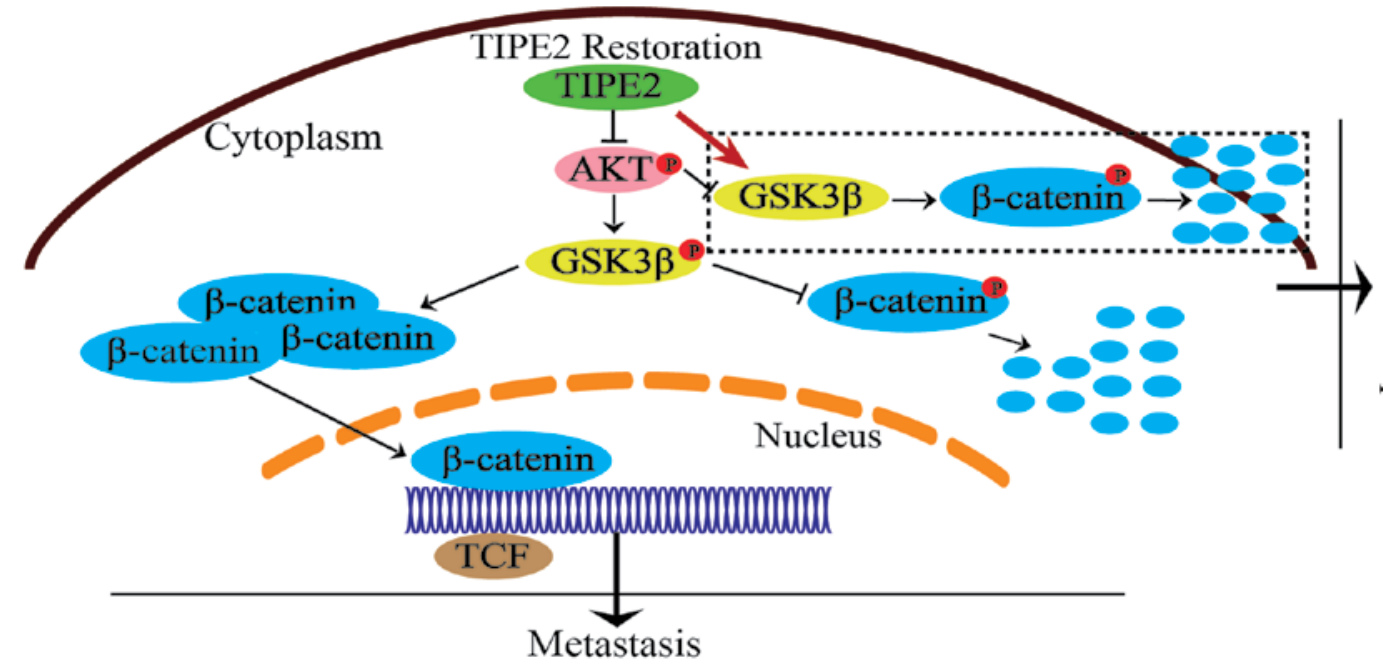

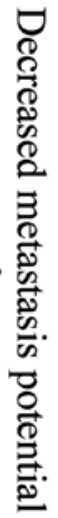

Figure 4. Schematic diagram of the antimetastatic activity of TIPE2 in gastric cancer. The loss of TIPE2 facilitates the activation of AKT signaling and phosphorylation/inactivation of GSK3 $\beta$ in gastric cancer. The inactivation of GSK3 $\beta$ results in the accumulation of $\beta$-catenin in the cytoplasm, enhancement of its translocation to nucleus and upregulation of $\beta$-catenin signaling, promoting gastric cancer metastasis. Adenovirus-mediated TIPE2 restoration promotes the degradation of $\beta$-catenin and downregulates the $\beta$-catenin signaling via inhibiting AKT and activating GSK3 $\beta$, leading to the inhibition of gastric cancer metastasis. 
showed that AdVTIPE2 promoted $\beta$-catenin degradation and decreased its translocation into nucleus in AGS tumor cells. The data indicated that TIPE2 restoration suppresses gastric cancer metastasis very possibly via downregulating $\beta$-catenin signaling through inhibiting AKT and activating GSK3 $\beta$ (Fig. 4).

\section{Discussion}

TIPE2 was initially found to be a crucial regulator of homeostasis maintenance $(1,3)$. Recently, TIPE2 has been shown to be frequently and remarkably reduced or lost in human hepatocellular, gastric and lung cancer tissues (8-12) as well as in most human cancer cell lines (17). Increasing evidence has revealed that TIPE2 exerts tumor-suppressive activity in several tumor types (8-12), implying it might be proposed as a novel candidate tumor suppressor gene. However, its tumor-suppressive effects and relevant mechanisms of its antitumor property have not been well characterized. In the present report, we investigated the significance of the TIPE2 in gastric cancer progression. We found that TIPE2 was almost absent in a panel of human gastric cancer cell lines compared with normal human gastric mucous epithelial cells, which is in accord with the clinical data (10). Forced expression of TIPE2 by adenovirus-mediated human TIPE2 (AdVTIPE2) gene transfer markedly suppressed gastric cancer migration and invasion. A previous study showed that TIPE2-stably transfected gastric cancer cells using eukaryotic expression plasmid displays similar migratory ability compared to control cells (10). One plausible explanation for the discrepancy is the different expression systems used in these studies. Adenovirusmediated transient overexpression in our study may avoid the persistent selection pressure of gastric cancer cells poisoned by TIPE2 that occurred during establishment of TIPE2-stably transfected tumor cells, thus providing more authentic data on tumor-suppressive effects of TIPE2 in gastric cancer.

$\beta$-catenin is a critical regulatory molecule of canonical Wnt signaling pathway $(20,21)$ and is also crucial for cadherinbased cell-cell adhesion by linking cadherins to actin cytoskeleton indirectly through $\alpha$-catenin $(22,23)$, which plays an important role in regulation of diverse cellular processes such as cell proliferation, survival, migration, invasion, polarity, differentiation, development and stem cell selfrenewal. Aberrant $\beta$-catenin signaling underlies a wide range of human diseases (21). $\beta$-catenin is generally considered as an oncogene that can facilitate cancer progression and metastasis (20). Upregulation of $\beta$-catenin signaling by its deregulation or mutational activation has been shown in various human cancers including gastric cancer $(20,24)$. The aberrant activation of PI3K/AKT signaling as well as the high inactivation of GSK $3 \beta$ is frequently found in human cancers $(25,26)$. It has been reported that activated AKT promotes the accumulation of cytosolic/nuclear $\beta$-catenin and enhances its transcriptional activity via directly phosphorylating $\beta$-catenin (27). GSK3 $\beta$ has been recognized as a primary kinase in the $\beta$-catenin multiprotein destruction complex containing axis inhibition protein (Axin), adenomatous polyposis coli (APC), GSK3 $\beta$ and casein kinase $1 \alpha(\mathrm{CK} 1 \alpha)$, which can phosphorylate $\beta$-catenin on conserved serine and threonine residues in its amino terminus (20). The phosphorylated $\beta$-catenin by GSK $3 \beta$ was then recog- nized by an E3 ubiquitin ligase $\beta$-transducin repeat-containing protein $(\beta \operatorname{TrCP})$, resulting in proteasomal degradation of $\beta$-catenin and low cytosolic/nuclear $\beta$-catenin levels (20). It has also been shown that AKT indirectly stabilizes $\beta$-catenin and consequently increases $\beta$-catenin signaling by phosphorylating GSK $3 \beta$ at Ser9 residue and inactivating GSK3 $\beta$ kinase activity (28). Thus, in addition to the Wnt-dependent pathway involved in modulation of $\beta$-catenin, the Wnt-independent pathway such as PI3K/AKT/GSK3 $\beta$ signaling pathway also critically activates transcriptional activity of $\beta$-catenin in many types of human cancer. Previous studies $(5,8)$ demonstrated that TIPE2 can inhibit AKT signaling via targeting RGL-PDK1-AKT and PI3K-Rac pathways. These results made us assume that TIPE2 suppressed gastric cancer cell migration and invasion possibly via downregulating $\beta$-catenin through activating GSK3 $\beta$ by attenuating AKT signaling. As expected, western blot and confocal fluorescence microscopic analysis showed that adenovirus-mediated TIPE2 overexpression remarkably downregulated pAKT, pGSK3 $\beta$ and total or nuclear $\beta$-catenin levels in human gastric cancer cells. Our present study indicated that TIPE2 restoration-mediated inhibition of AKT activation might result in the decreased phosphorylation of GSK $3 \beta$ and increased active GSK3 $\beta$, leading to the phosphorylation and degradation of $\beta$-catenin through a ubiquitin-dependent proteasome pathway. The TIPE2/AKT/GSK3 $\beta / \beta$-catenin axis may constitute a major pathway to regulate gastric cancer metastasis.

Collectively, we demonstrated that TIPE2 was absent in human gastric cancer cells. Adenovirus-mediated TIPE2 restoration markedly suppressed gastric cancer cell migration and invasion possibly via inhibiting $\beta$-catenin signaling through suppressing AKT as well as activating GSK3 $\beta$. Our data provided the first evidence that TIPE2 may be a potential therapeutic target for human gastric cancer metastasis.

\section{Acknowledgements}

This study was supported by grants from the National Natural Science Foundation of China (NNSFC) (nos. 81372443, 81001016,81272542 and 81572992) and the Science and Technology Department of Jiangsu Province (nos. BL2014039 and BY2015039).

\section{References}

1. Sun H, Gong S, Carmody RJ, Hilliard A, Li L, Sun J, Kong L, $\mathrm{Xu} \mathrm{L}$, Hilliard B, Hu S, et al: TIPE2, a negative regulator of innate and adaptive immunity that maintains immune homeostasis. Cell 133: 415-426, 2008.

2. Lou Y and Liu S: The TIPE (TNFAIP8) family in inflammation, immunity, and cancer. Mol Immunol 49: 4-7, 2011.

3. Freundt EC, Bidere $\mathrm{N}$ and Lenardo MJ: A different TIPE of immune homeostasis. Cell 133: 401-402, 2008.

4. Wang Z, Fayngerts S, Wang P, Sun H, Johnson DS, Ruan Q, Guo W and Chen YH: TIPE2 protein serves as a negative regulator of phagocytosis and oxidative burst during infection. Proc Natl Acad Sci USA 109: 15413-15418, 2012.

5. Sun H, Zhuang G, Chai L, Wang Z, Johnson D, Ma Y and Chen YH: TIPE2 controls innate immunity to RNA by targeting the phosphatidylinositol 3-kinase-Rac pathway. J Immunol 189: 2768-2773, 2012.

6. Lou Y, Zhang G, Geng M, Zhang W, Cui J and Liu S: TIPE2 negatively regulates inflammation by switching arginine metabolism from nitric oxide synthase to arginase. PLoS One 9: e96508, 2014. 
7. Luan YY, Yao YM, Zhang L, Dong N, Zhang QH, Yu Y and Sheng ZY: Expression of tumor necrosis factor- $\alpha$ induced protein 8 like-2 contributes to the immunosuppressive property of CD4(+)CD25(+) regulatory T cells in mice. Mol Immunol 49: 219-226, 2011

8. Gus-Brautbar Y, Johnson D, Zhang L, Sun H, Wang P, Zhang S, Zhang L and Chen YH: The anti-inflammatory TIPE2 is an inhibitor of the oncogenic Ras. Mol Cell 45: 610-618, 2012.

9. Cao X, Zhang L, Shi Y, Sun Y, Dai S, Guo C, Zhu F, Wang Q, Wang J, Wang X, et al: Human tumor necrosis factor (TNF)alpha-induced protein 8-like 2 suppresses hepatocellular carcinoma metastasis through inhibiting Rac1. Mol Cancer 12: 149, 2013.

10. Zhao Q, Zhao M, Dong T, Zhou C, Peng Y, Zhou X, Fan B Ma W, Han M and Liu S: Tumor necrosis factor- $\alpha$-induced protein-8 like-2 (TIPE2) upregulates p27 to decrease gastic cancer cell proliferation. J Cell Biochem 116: 1121-1129, 2015

11. Liu QQ, Zhang FF, Wang F, Qiu JH, Luo CH, Zhu GY and Liu YF: TIPE2 inhibits lung cancer growth attributing to promotion of apoptosis by regulating some apoptotic molecules expression. PLoS One 10: e0126176, 2015.

12. Li Y, Li X, Liu G, Sun R, Wang L, Wang J and Wang H: Downregulated TIPE2 is associated with poor prognosis and promotes cell proliferation in non-small cell lung cancer. Biochem Biophys Res Commun 457: 43-49, 2015.

13. Jemal A, Bray F, Center MM, Ferlay J, Ward E and Forman D Global cancer statistics. CA Cancer J Clin 61: 69-90, 2011.

14. He TC, Zhou S, da Costa LT, Yu J, Kinzler KW and Vogelstein B: A simplified system for generating recombinant adenoviruses. Proc Natl Acad Sci USA 95: 2509-2514, 1998.

15. Liu Y, Ye T, Sun D, Maynard J and Deisseroth A: Conditionally replication-competent adenoviral vectors with enhanced infectivity for use in gene therapy of melanoma. Hum Gene Ther 15 637-647, 2004

16. Xie Y, Sheng W, Xiang J, Ye Z and Yang J: Interleukin-17F suppresses hepatocarcinoma cell growth via inhibition of tumor angiogenesis. Cancer Invest 28: 598-607, 2010.
17. Zhang G, Hao C, Lou Y, Xi W, Wang X, Wang Y, Qu Z, Guo C, Chen $Y$ and Zhang Y: Tissue-specific expression of TIPE2 provides insights into its function. Mol Immunol 47: 2435-2442, 2010.

18. Hanahan D and Weinberg RA: Hallmarks of cancer: The next generation. Cell 144: 646-674, 2011.

19. Chaffer CL and Weinberg RA: A perspective on cancer cell metastasis. Science 331: 1559-1564, 2011.

20. Anastas JN and Moon RT: WNT signalling pathways as therapeutic targets in cancer. Nat Rev Cancer 13: 11-26, 2013.

21. Clevers $\mathrm{H}$ and Nusse R: Wnt/ $\beta$-catenin signaling and disease. Cell 149: 1192-1205, 2012.

22. Conacci-Sorrell M,Zhurinsky J and Ben-Ze'ev A: The cadherincatenin adhesion system in signaling and cancer. J Clin Invest 109: 987-991, 2002.

23. Nelson WJ and Nusse R: Convergence of Wnt, beta-catenin, and cadherin pathways. Science 303: 1483-1487, 2004.

24. Chiurillo MA: Role of the Wnt/ $\beta$-catenin pathway in gastric cancer: An in-depth literature review. World J Exp Med 5: 84-102, 2015.

25. Porta C, Paglino C and Mosca A: Targeting PI3K/Akt/mTOR Signaling in Cancer. Front Oncol 4: 64, 2014.

26. Kang T, Wei Y, Honaker Y, Yamaguchi H, Appella E, Hung MC and Piwnica-Worms H: GSK-3 beta targets Cdc25A for ubiquitinmediated proteolysis, and GSK-3 beta inactivation correlates with Cdc25A overproduction in human cancers. Cancer Cell 13 36-47, 2008.

27. Fang D, Hawke D, Zheng Y, Xia Y, Meisenhelder J, Nika H, Mills GB, Kobayashi R, Hunter T and Lu Z: Phosphorylation of beta-catenin by AKT promotes beta-catenin transcriptional activity. J Biol Chem 282: 11221-11229, 2007.

28. Cohen P and Frame S: The renaissance of GSK3. Nat Rev Mol Cell Biol 2: 769-776, 2001. 